Czerepinski, PJ., Nix, AR., Tariq, MF., Bull, DR., \& Canagarajah, CN. (2000). Robust and scalable matching pursuits video transmission using the Bluetooth air interface standard. IEEE Transactions on Vehicular Technology, 46(3), 673 - 681.

https://doi.org/10.1109/30.883429, https://doi.org/10.1109/ICCE.2000.854643

Peer reviewed version

Link to published version (if available):

10.1109/30.883429

10.1109/ICCE.2000.854643

Link to publication record in Explore Bristol Research

PDF-document

University of Bristol - Explore Bristol Research

General rights

This document is made available in accordance with publisher policies. Please cite only the published version using the reference above. Full terms of use are available:

http://www.bristol.ac.uk/red/research-policy/pure/user-guides/ebr-terms/ 


\title{
ROBUST AND SCALABLE MATCHING PURSUITS VIDEO TRANSMISSION USING THE BLUETOOTH AIR INTERFACE STANDARD
}

\author{
M. Fahim Tariq, Przemystaw Czerepiński, Andrew Nix, David Bull and Nishan Canagarajah \\ Centre for Communications Research, University of Bristol \\ Merchant Venturers Bldg., Woodland Rd., Bristol BS8 1UB, United Kingdom \\ email: \{M.F.Tariq, P.J.Czerepinski\}@bristol.ac.uk
}

\begin{abstract}
This paper introduces an error resilient implementation of the matching pursuits algorithm for video coding. The video bitstream is transmitted using the simulated Bluetooth air interface standard, which recommends $A R Q$ as a means of overcoming channel errors in data packets. This may be unsuitable for real time and broadcast applications. Therefore, a modified receiver is proposed in this paper, which does not request a retransmission of erroneous packets, but instead passes them to the video decoder to exploit error resilience. This strategy is shown to be superior to a standard compliant system if $A R Q$ cannot be applied. This work confirms that wireless communication standards should support a transparent mode for video applications.
\end{abstract}

\section{INTRODUCTION}

Bluetooth/1 is a short-range radio data communication standard released by ETSI in July 1999 [1]. It can be used as a data access point, a cable replacement or to form an ad-hoc network between electronic devices. The aim of this paper is to investigate the implications of transmitting real time video using the Bluetooth/1 standard.

Since the majority of video coding algorithms employ a combination of predictive and variable length coding (VLC), they are known to fail catastrophically in the presence of channel errors. Techniques such as forward error correction (FEC) and retransmission (ARQ) are often employed to overcome this problem. However, the former can compromise the compression performance, especially if transmission across a time varying channel is required, while the latter is usually unsuitable for real time or broadcast applications. Error resilient techniques are attractive bearers of multimedia information across noisy environments as they tolerate a certain level of transmission error and provide acceptable quality of reconstruction without resorting to FEC or $A R Q$.

The contribution of this paper is twofold: Firstly, to introduce a scalable and error resilient implementation of a state-of-the art video codec, known as the matching pursuits algorithm. Secondly, to characterize the transmission of the video bitstream over a wireless network, using the ETSI Bluetooth/1 standard [1]. The standard recommends a low-level rejection of erroneous data packets. In simulcast mode, such packets are retransmitted, which increases the delay. In broadcast mode they are discarded at the receiver by means of the flushing procedure. In this paper, a modification is considered to make such packets available to the error resilient video decoder. This strategy is demonstrated to provide a better quality of service, compared to the mandatory discarding of the corrupted packets.

\section{BLUETOOTH/1 SIMULATION MODEL}

Bluetooth $/ 1$ is a time division duplex (TDD) system that operates in the unlicensed ISM band at $2.4 \mathrm{GHz}$ with slow frequency hopping (to combat interference and fading) at a rate of 1600 hops/s. The gross bit rate is $1 \mathrm{Mbps}$ within a $1 \mathrm{MHz}$ channel bandwidth on a GFSK modulated carrier with $\mathrm{BT}=0.5$ and modulation index in the range 0.28 0.38 [1]. Figure 1 shows the block diagram of the Bluetooth 1 simulation model, and Figure 2 shows the data transmission results. An independent, uncorrelated Rayleigh faded, static channel was assumed for every packet. It can be verified from Figure 2 that a value of $\mathrm{Eb} / \mathrm{No}$ equal to $40 \mathrm{~dB}$ is required to obtain a 1 in 10,000 bit error rate. This corresponds to a packet error rate of less than $1 \%$.

The transmitter generates the cyclic redundancy check (CRC) using an arbitrary Bluetooth device Upper Address Part (UAP), equal to 40 (hex). Should a received packet fail the CRC, the receiver does not request retransmission, due to low-delay constraints imposed by real-time video communication. Instead, the user data are passed to the error resilient video decoder. This procedure is used for both simulcast and broadcast transmission.

\section{THE VIDEO CODEC}

The video codec is based on matching pursuits [2]. For maximum compaction, video is normally variable-length coded. However, the codec presented in this paper is

This work was supported by the EPSRC grant GR-L43596. 


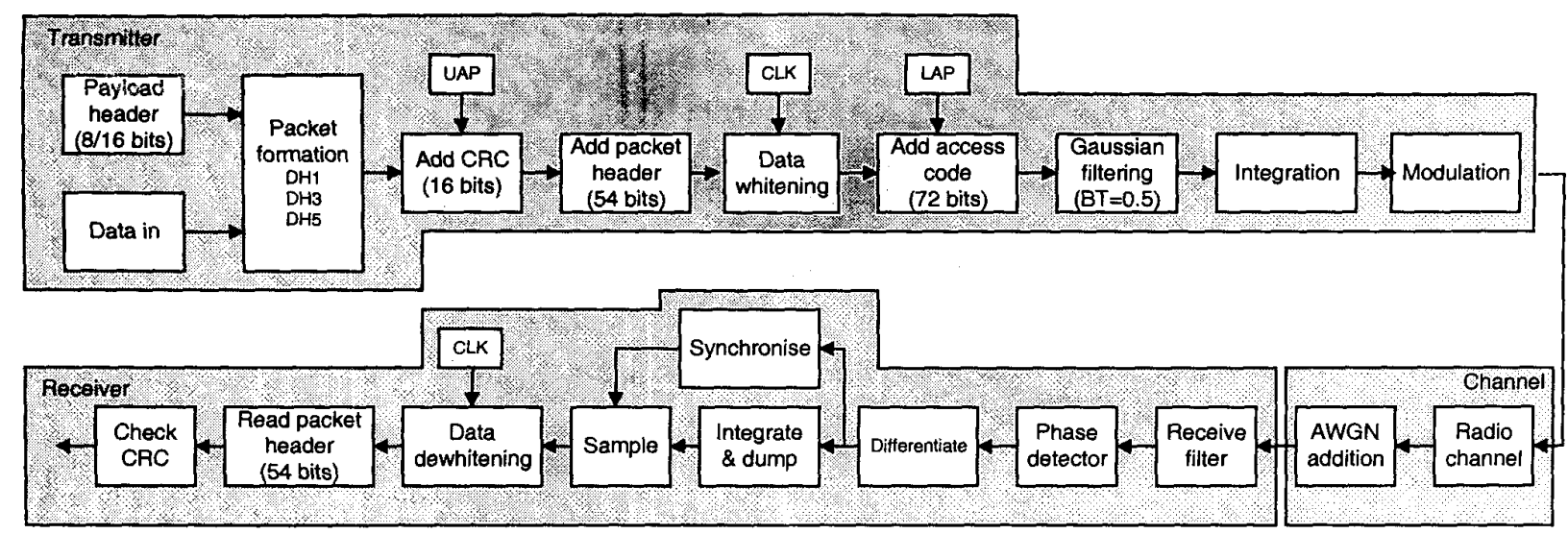

Figure 1. Block diagram of the Bluetooth/1 system.

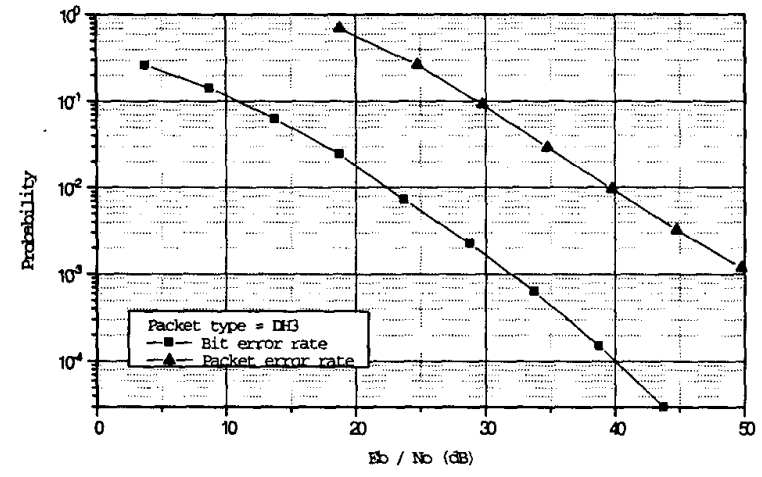

Figure 2. Raw bit error rate and packet error rate of Bluetooth/1 in an uncorrelated, static Rayleigh faded channel.

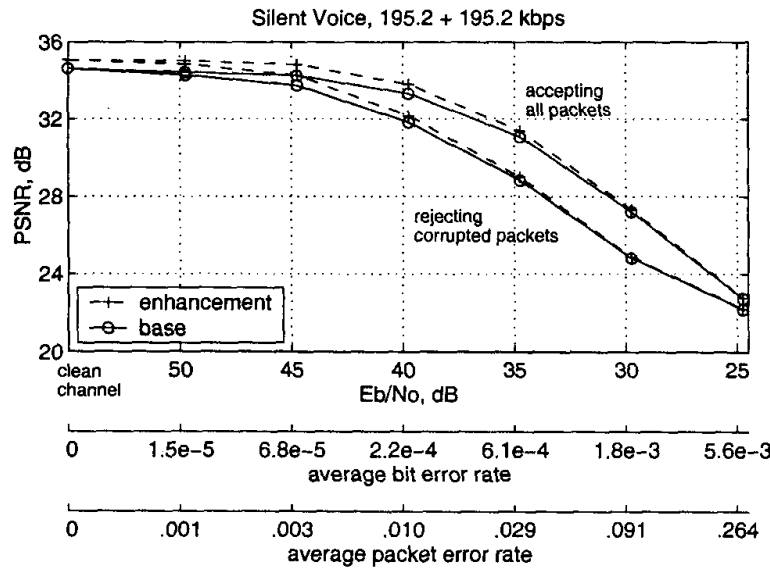

Figure 3. The PSNR performance of the proposed codec in a noisy Bluetooth $/ 1$ environment.

designed for error resilience, and therefore VLC is not employed to avoid a catastrophic failure in the presence of channel errors. Matching pursuits compression involves positional coding, which was accomplished using the Error Resilient Positional Code (ERPC) [3]. It should be noted that a small portion of the data, such as the frame type, the temporal reference and the quantization parameters is highly protected against channel errors by means of forward error correction. These data fields are crucial for any meaningful decoding and constitute a small enough fraction of the bitstream to not compromise the compression performance.

A two-layer SNR scalable codec was implemented. The base layer encoder operates in a standard motion compensated configuration. The enhancement layer adopts the reconstructed base layer signal as a prediction. This strategy prevents any temporal error propagation in the enhancement layer. The base layer can be decoded independently of the enhancement layer to provide a lower quality reconstructed signal. Decoding both layers provides a higher quality reconstructed signal.

\section{RESULTS AND CONCLUSION}

The performance of the proposed video codec was tested in the presence of channel errors. Bluetooth/1 packet type DH3 was selected, which offers a symmetric user data rate equal to $390.4 \mathrm{kbps}$ with no forward error correction. The sequence 'Silent Voice' was coded to match this bit rate, with equal rates of $195.2 \mathrm{kbps}$ allocated to base and enhancement layers. It can be verified from Figure 3 that the proposed approach, which allows the error resilient decoder to utilize all (also erroneous) packets, outperforms the standard compliant system which rejects corrupted packets. This result is a strong case for Bluetooth/1 and other wireless data standards to support a transparent mode for video applications.

\section{REFERENCES}

[1] Bluetooth System Specification version 1.0, December 1999.

[2] Neff R. and Zakhor A. Very Low Bit-Rate Video Coding Based on Matching Pursuits. IEEE Trans. CSVT, vol. 7, pp. 158-171, February 1997.

[3] Cheng N.-T and Kingsbury N.G. The ERPC: An Efficient Error-Resilient Technique for Encoding Positional Information or Sparse Data. IEEE Trans. COM, vol. 40, pp. 140-148, January 1992. 